tion of functions of the body. The argument was that these organs and functions have to be nearly perfect or else they are worse than useless to the organism. However, this view is now challenged by perhaps most forcefully by Richard Dawkins, who in The Blind Watchmaker (Longman, 1986) claims that evolutionary change can explain all.

The final chapter, modestly described by the author as a "postscript", deals with a few twentieth-century developments such as Freud's 'explanations' of religion, the revolution in physics and the majority of evolutionary biologists,

the resurgence of the interest in science and human values. Last, but not least, is the "Bibliographic Essay" which fills more than 50 pages.

Brooke's vast reading and the impressive use he has made of it attest to the complexity of the relationship between science and religion. To use his words, there is no such thing as the relationship, whether it is couched in terms of war or of peace.

H. N. V. Temperley is at Thorney House, Thorney, Langport, Somerset TA10 ODW, UK.

\section{Preparing for the brain decade}

\author{
Michael R. Hanley
}

Neurons and Networks: An Introduction to Neuroscience. By John E. Dowling. Belknap (Harvard University Press): 1992. Pp. 447. \$45, £29.95.

The Neuron: Cell and Molecular Biology. By Irwin B. Levitan and Leonard K. Kaczmarek. Oxford University Press: 1991. Pp. 450. £55, $\$ 65$ (hbk); $£ 18.95, \$ 39.95$ (pbk).

An Introduction to Molecular Neurobiology. Edited by Zach W. Hall. W. H. Freeman (UK)/Sinauer (US): 1992. Pp. 555. £32.95, \$46.95.

THE phrase 'coming of age' is used in two of the three introductions to these new neurobiology textbooks in recognition that the discipline has, in many senses, grown into adulthood after a protracted adolescence. Firm foundations of hard facts now exist in specialist fields of neurobiology, such as the molecular mechanisms of neural development where previously there were black boxes. Moreover, technical innovation, such as patch clamping and hybridoma technology, has made accessible previously intractable problems, such as the structural analysis of excitability proteins. It now seems that there is a cohesive core of knowledge about techniques and principles of neurobiology; and each of these texts takes a different aspect of this knowledge as its starting point. All three approaches work well, and the textbooks are uniformly excellent.

Neurons and Networks covers the classic material of neurobiology. It has evolved from years of lectures given to undergraduates at Harvard University by the renowned retinal physiologist John Dowling. The text is divided into two sections, "Cellular Neuroscience" and "Integrative Neuroscience", but the author has wisely focused on his own specialist area, the visual system, to develop concepts throughout the book.
Parts of the first section are weak. Specifically, the chapter on the chemistry of synaptic transmission is not as up to date as the corresponding material in the other two books. A telling gaffe is the repeated use of "inositol triphosphate" - a nonexistent compound - for inositol trisphosphate (my italics). The second section on integrative neuroscience is built on an evolutionary progression from invertebrates to vertebrates to tervening chapter on developmental neurobiology. Overall, this is a sober and scholarly volume that strikes me, with its terse, no-frills style and reliance on the history of ideas, as very Harvard. Although intended as an introduction to basic neurobiology, it is a satisfying book at several levels.

The Neuron is a more advanced presentation, focusing on cell and molecular neurobiology at the level of graduate students. The authors, Irwin Levitan and Leonard Kaczmarek, are distinguished neuroscientists with a history of various types of collaborations in teaching, writing and research. In writing together, they have evolved a distinctive style, which is often slyly irreverent. The text is constructed from three sections: "Electrical Properties of Neurons", "Intercellular Communication" and "Behavior and Plasticity". The text is impressively modern, with up-to-date information on the trendiest areas of neurobiology, such as adhesion molecules, synaptic vesicle biogenesis and function, and ion channel systems. Much of this new material is unavailable in comparable texts. The book is highly visual, with figures on virtually every page. The figures deserve special comment because they are a teacher's dream: simple and uncluttered, but conceptually powerful. Frankly, although the recommendation is often abused, The Neuron is one of those books that really do belong on every shelf. higher cognitive functions, with an in-
Introduction to Molecular Neurobiology is a multi-author volume edited by another distinguished neurobiologist, Zach Hall, and draws heavily on the editorial ranks of the journal Neuron. It is the most detailed and sophisticated of the three books. The chapters are organized as four sections: "Signalling in the Nervous System", "Cell Biology of Neurons and Glia", "Neuronal Development" and "Complex Interactions of Neurons and Neuronal Disorders". Because of its explicit focus on molecular aspects, this text includes extensive treatment of topics not found in the other two volumes, such as the molecular basis of developmental neurobiology and the genetics of neurological disease. The book is rich with figures and often uses inserts to introduce a self-contained discussion of a single subject, such as video microscopy, at a point where the subject is relevant but distracting if presented in the main text. Indeed, the visual presentation is so good that the text almost seems to be a giant figure legend. The book provides the comprehensive treatment of molecular neurobiology that has been sorely needed. I regard it as the definitive specialist text of the subject, and it is likely to remain so for some time, given the high standard of the contributions.

Lastly, there are important similarities between the texts which contribute to their outstanding quality as educational aids. They all present a summary at the end of each chapter, which restates the important generalizations that emerge from the details of specific experiments. Each book also recommends a few key references, emphasizing recent reviews and pivotal papers.

The expansion of neurobiology has led to greater integration of molecular, physiological, anatomical and systems studies than ever before, but has also created an unprecedented volume of information. The complete neurobiologist has to be familiar with a huge diversity of subjects from genetics to psychophysics. So is it wise, or even possible, to cover the spectrum of subjects now subsumed by neurobiology in a single volume? These books have not attempted to do so; rather, they have each defined a specific orientation that cuts across the main issues in neurobiology. They are not in competition, but complement each other, and all could be used effectively in a modern neurobiology curriculum. And researchers should consider adding them to personal libraries, as they mark the emergence of a new generation of modern neuroscience texts.

Michael R. Hanley is in the Department of Biological Chemistry, School of Medicine, University of California, Davis, California 95616, USA.

NATURE · VOL 359 • 22 OCTOBER 1992 